Article

\title{
Efficiency for Vector Variational Quotient Problems with Curvilinear Integrals on Riemannian Manifolds via Geodesic Quasiinvexity
}

\author{
Tiziana Ciano ${ }^{1,2,+} \mathbb{C}^{\mathbb{C}}$, Massimiliano Ferrara ${ }^{1,3}{ }^{\mathbb{C}}$, Ştefan Mititelu ${ }^{4}$ and Bruno Antonio Pansera ${ }^{1, *}$ \\ 1 Department of Law, Economics and Human Sciences \& Decisions_Lab, University “Mediterranea" of Reggio \\ Calabria, via dell’Universitá, 25, I-89124 Reggio Calabria, Italy; tiziana.ciano@unirc.it (T.C.); \\ massimiliano.ferrara@unirc.it (M.F.) \\ 2 Faculty of Business and Law, School of Law Richmond Bulding Portland Street, University of Portsmouth, \\ Portsmouth P013DE, UK \\ 3 Department of Management and Technology, ICRIOS-The Invernizzi Centre for Research in Innovation, \\ Organization, Strategy and Entrepreneurship Bocconi University, via Sarfatti, 25, I-20136 Milano, Italy \\ 4 Department of Mathematics and Informatics, University of Bucharest, 010014 Bucharest, Romania; \\ direttore.digies@unirc.it \\ * Correspondence: bruno.pansera@unirc.it \\ + These authors contributed equally to this work.
}

Received: 25 May 2020; Accepted: 24 June 2020; Published: 30 June 2020

\begin{abstract}
In the paper, we analyze the necessary efficiency conditions for scalar, vectorial and vector fractional variational problems using curvilinear integrals as objectives and we establish sufficient conditions of efficiency to the above variational problems. The efficiency sufficient conditions use of notions of the geodesic invex set and of (strictly, monotonic) $(\rho, b)$-geodesic quasiinvex functions.
\end{abstract}

Keywords: curvilinear integrals; geodesic quasiinvexity; Riemannian manifolds; efficient solution

\section{Introduction and Preliminaries}

\subsection{Aim of the Work}

Valentine [1] began the study of scalar variational problems with constraints by establishing the necessary conditions of optimality in 1937. Later, Mond and Hanson in 1967 [2], Mond, Chandra and Husain in 1988 [3], Mond and Husain in 1989 [4], Preda [5] and others developed different aspects of duality for scalar variational problems using optimality conditions. Using the techniques of Chankong-Haimes [6] and Kanniappan [7], Mukerjee and Purnachandra Rao [8] developed duality results, and Preda and Gramatovici [9] proved the sufficient optimality conditions for multiobjective variational problems. Using Jaganatan's methods [10], Mititelu et al. [11-18] established the necessary efficiency conditions and duality conditions for multiobjective fractional variational problems on the basis of the notion of $(\rho, b)$-quasiinvexity. Kim and Kim [19] used the efficiency property of nondifferentiable multiobjective variational problems in duality theory under generalized convexity assumptions.

\subsection{Preliminaries, Tools and Definitions}

Inspired by these aspects of the scalar variational problems, Udrişte et al. [16,17,20-23], proceeded to the optimality study of variational problems in many dimensions (multitime variational problems) with constraints, using multiple integrals and curvilinear integrals. In this work, we establish the necessary efficiency conditions under new forms, and we prove the sufficient efficiency conditions for 
these problems. The sufficient conditions of efficiency are based on the notions of the geodesic invex set and (strictly) $(\rho, b)$-geodesic quasiinvex functions on Riemannian manifolds.

Let $(T, h)$ and $(M, g)$ be Riemannian manifolds of dimensions $p$ and $n$, respectively. The local coordinates on $T$ and $M$ are written as $t=\left(t^{k}\right), t=1, \ldots, p$ and $x=\left(x^{i}\right), i=1, \ldots, n$, respectively. Let $J^{1}(T, M)$ be the first-order jet bundle associated with $T$ and $M$, from which we hold $x: T \rightarrow M, x=$ $x(t), t \in T$. Let $\Omega$ be a measurable set in $T$, the different points $t_{1}=\left(t_{1}^{1}, \ldots, t_{1}^{p}\right), t_{2}=\left(t_{2}^{1}, \ldots, t_{2}^{p}\right) \in \Omega$ and suppose that $C: t=t(\tau), \tau \in[a, b]$ (or $t \in\left[t_{1} t_{2}\right]$ ) is a piecewise $C^{1}$-class curve joining the points $t_{1}$ and $t_{2}$ in $\Omega$. Then $x(C) \subset M$. We consider the functions

$$
\begin{aligned}
& g=\left(g_{\beta}\right): J^{1}(T, M) \rightarrow R^{m}, \quad \beta=1, \ldots, m, \\
& h=\left(h_{\kappa}\right): J^{1}(T, M) \rightarrow R^{q}, \kappa=1, \ldots, q,
\end{aligned}
$$

and for $\alpha=1, \ldots, p$, we consider the function:

$$
\begin{gathered}
s_{\alpha}: J^{1}(T, M) \rightarrow R, X_{\alpha}: J^{1}(T, M) \rightarrow R \\
f_{\alpha}=f_{\alpha}^{r}: J^{1}(T, M) \rightarrow R, \kappa_{\alpha}=\kappa_{\alpha}^{r}: J^{1}(T, M) \rightarrow R, r=1, \ldots, p .
\end{gathered}
$$

The arguments of these functions are $j^{1} x=\left(t, x, x_{0}\right)$, the first prolongation jet of $x$. For the functions $x, s_{\alpha}, f_{\alpha}, g_{\beta}$ and $h_{\kappa}$ we use the pullback $j_{t}^{1} x=\left(j^{1} x\right)(t)=\left(t, x(t), x_{v}(t)\right)$, where $t \in \Omega$, $x(t)=\left(x^{k}(t)\right)$ and $\left(\partial x / \partial t^{v}(t)\right)=\left(x_{v}(t)\right)=\left(x_{v}^{i}(t)\right)$.

We suppose that $x, s_{\alpha}, f_{\alpha}, \kappa_{\alpha}, g, h \in C^{2}(\Omega)$ and that $M$ is a complete manifold. In this paper we use the notation $D_{v}=\frac{\partial}{\partial t^{v}}(v=1, \ldots, p)$ for the total derivative. Additionally, we use the norm $\|x\|=\|x\|_{\infty}+\sum_{j=1}^{n}\left\|x^{j}\right\|_{\infty}$ and denote

$$
F(\Omega, M)=\{x: \Omega \rightarrow M \mid x(\cdot) \text { is normed and piecewise continuous on } \Omega\},
$$

where, through $x(\cdot)$, we denote the function $x: \Omega \rightarrow M$.

Throughout this paper, for two vectors $v=\left(v_{1}, \ldots, v_{n}\right)$ and $w=\left(w_{1}, \ldots, w_{n}\right)$ the relations of the form $v=w, v<w, v \leq w, v \leq w$ are defined as follows

$$
\begin{aligned}
& v=w \Leftrightarrow v_{i}=w_{i}, i=1, \ldots, n ; v<w \Leftrightarrow v_{i}<w_{i}, i=1, \ldots, n ; \\
& v \leq w \Leftrightarrow v_{i} \leq w_{i}, i=1, \ldots, n ; v<w \Leftrightarrow v \leq w \text { and } v \neq w .
\end{aligned}
$$

The purpose of this paper is to give the optimality conditions for multitime variational problems with objectives of the following forms:

$$
\begin{gathered}
S(x(\cdot))=\int_{C} s_{\alpha}\left(j_{t}^{1} x\right) d t^{\alpha}, \\
\left(\int_{C} f_{\alpha}^{1}\left(j_{t}^{1} x\right) d t^{\alpha}, \ldots, \int_{C} f_{\alpha}^{p}\left(j_{t}^{1} x\right) d t^{\alpha}\right), \\
\left(\frac{\int_{C} f_{\alpha}^{1}\left(j_{t}^{1} x\right) d t^{\alpha}}{\int_{C} \kappa_{\alpha}^{1}\left(j_{t}^{1} x\right) d t^{\alpha}}, \ldots, \frac{\int_{C} f_{\alpha}^{p}\left(j_{t}^{1} x\right) d t^{\alpha}}{\int_{C} \kappa_{\alpha}^{p}\left(j_{t}^{1} x\right) d t^{\alpha}}\right),
\end{gathered}
$$

where

$$
\int_{C} \kappa_{\alpha}^{r}\left(j_{t}^{1} x\right) d t^{\alpha}>0 \text { for } r=1, \ldots, p .
$$

As usual, the functionals of mechanical work type, due to their physical meaning, or similarly, the cost functionals in economics, are very important in applications. Thus, in our opinion, the 
centrality of the present work is supported by both theoretical and practical reasoning. As well, the ideas and techniques of this paper may stimulate further research in this dynamic field. The paper was organized as follows. Section 1 (structured in two sub-sections) is an introduction presenting the aim of the study and the technical tools useful for the sequel. Section 2 presents a scalar multi-time variational problem with constraints. An efficiency solution is defined and efficiency conditions for the program (SPV) are given. Section 3, contains necessary conditions for a vector curvilinear program establishing a Pareto minimum point. In the Section 4, necessary conditions for the quotient variational curvilinear problem are presented. This case study is particularly strategical for potential applications in the frame of multiobjective programming. Sections 5 and 6 condense sufficient efficiency conditions related to the classes of problems precedently introduced. The paper, in the last section, contains the conclusions and potential further developments.

\subsection{Geodesic Invex Set and $(\rho, b)$-Geodesic Quasiinvex Functionals}

In [24], Barani and Pouryayaei introduced the notions of the invex set and invex function in the following ways.

Definition 1 ([24]). Let $(M, g)$ be a complete Riemannian manifold. Let $\eta: M \times M \rightarrow T M, \eta(u, x) \in T_{x} M$, $u, x \in M$ be a vector function and $S \subset M$ be a nonempty set.

(i) The set $S$ is called $\eta$-geodesic invex if, for every $u, x \in S$, there exists exactly one geodesic $\gamma_{u, x}:[0,1] \rightarrow$ M such that

$$
\gamma_{u, x}(0)=u, \dot{\gamma}_{u, x}(0)=\eta(u, x), \gamma_{u, x}(\theta) \in S, \forall \theta \in[0,1] .
$$

Example 1. $\gamma_{u, x}(t)=u+t \eta(x, u)$. For examples of geodesic invex sets, see [24].

(ii) Let $S \subset M$ be an open $\eta$-geodesic invex set and $f: S \rightarrow R$ be a $C^{1}$ function. The function $f$ is called $\eta$-geodesic invex on $S$ if

$$
f(x)-f(u) \geq d f_{u}(\eta(u, x)) .
$$

For examples of geodesic invex sets and geodesic invex functions, see [24].

Definition $2([18,20])$. Let $x^{0}(\cdot), x(\cdot) \in F(\Omega, M)$, where $x^{0}(\cdot)$ is fixed. A function $\varphi(t, \theta), t \in \Omega, \theta \in[0,1]$ is called a geodesic deformation of the pair of functions $x^{0}(\cdot), x(\cdot)$ if it satisfies the following properties:

(1) The function $\theta \rightarrow \varphi(t, \theta)$ is a geodesic.

(2) $\varphi(t, 0)=x^{0}(t), \varphi(t, 1)=x(t), \varphi(\cdot, \theta) \in S \subset F(\Omega, M), \forall \theta \in[0,1]$.

We say that $x(\cdot)$ is a geodesic deformation of $x^{0}(\cdot)$.

Definition 3. A set $G \subset F(\Omega, M)$ is called $\eta$-geodesic invex if, for every $x^{0}(\cdot), x(\cdot) \in G$, there exists exactly one geodesic deformation $\varphi(t, \theta), t \in \Omega, \theta \in[0,1]$ such that the vector function

$$
\eta(t)=\eta\left(x^{0}(t), x(t)\right)=\left(\eta^{1}(t), \ldots, \eta^{n}(t)\right)=\left.\frac{\partial \varphi}{\partial \theta}(t, \theta)\right|_{\theta=0} \in T_{x^{0}(t)} M
$$

is of class $C^{1}$ and satisfies $\eta\left(t_{1}\right)=0, \eta\left(t_{2}\right)=0$.

In order to get our sufficient conditions of efficiency, we shall introduce the notion of (monotonic) $(\rho, b)$-geodesic quasiinvex functionals.

We fix a number $\rho \in R$, a functional $b: F(\Omega, M) \times F(\Omega, M) \rightarrow[0, \infty)$ and the distance function $d(x(\cdot), y(\cdot))$ on $F(\Omega, M)$. We consider the following functional, useful for the sequel:

$$
E: F(\Omega, M) \rightarrow R, E(x(\cdot))=\int_{C} X_{\alpha}\left(t, x(t), x_{v}(t)\right) d t^{\alpha} .
$$


Definition 4. Let $(M, g)$ be a complete Riemannian manifold. Let $G$ be an open $\eta$-geodesic invex subset of $F(\Omega, M)$.

(i) The functional $E$ is called (strictly) $(\rho, b)$-geodesic quasiinvex at $x^{0}(\cdot) \in G$ with respect to $\eta(t)$ if $E(x) \leq E\left(x^{0}\right) \Rightarrow$

$$
\begin{gathered}
b\left(x, x^{0}\right) \int_{C}\left(\eta_{i} \frac{\partial X_{\alpha}}{\partial x^{i}}\left(t, x^{0}(t), x_{v}^{0}(t)\right)+\frac{\partial \eta_{i}}{\partial t^{v}} \frac{\partial X_{\alpha}}{\partial x_{v}^{i}}\left(t, x^{0}(t), x_{v}^{0}(t)\right)\right) d t^{\alpha}(<) \leq \\
\leq-\rho b\left(x, x^{0}\right) d^{2}\left(x, x^{0}\right),
\end{gathered}
$$

for any $x(\cdot) \in G$.

(ii) The functional $E$ is called monotonic $(\rho, b)$-geodesic quasiinvex at $x^{0}(\cdot) \in G$ with respect to $\eta(t)$ if $E(x)=E\left(x^{0}\right) \Rightarrow$

$$
\begin{gathered}
b\left(x, x^{0}\right) \int_{C}\left(\eta_{i} \frac{\partial X_{\alpha}}{\partial x^{i}}\left(t, x^{0}(t), x_{v}^{0}(t)\right)+\frac{\partial \eta_{i}}{\partial t^{v}} \frac{\partial X_{\alpha}}{\partial x_{v}^{i}}\left(t, x^{0}(t), x_{v}^{0}(t)\right)\right) d t^{\alpha}= \\
=-\rho b\left(x, x^{0}\right) d^{2}\left(x, x^{0}\right),
\end{gathered}
$$

for any $x(\cdot) \in G$.

Example 2. Let $a:[0,1]^{2} \times C^{\infty}[0,1] \rightarrow R$, the curve $C: t^{1}=\tau, t^{2}=\tau^{3}, \tau \in[0,1], t \in\left[t^{1}, t^{2}\right] \in \Omega=$ $[0,1]^{2}$ and the continuous function $x(\cdot)=\left(x^{1}(\cdot), x^{2}(\cdot)\right)$, or $x: \Omega \rightarrow M \subset R^{2}$, defined by $x=x(t), t \in \Omega$. We define the set $M=x(\Omega)=x\left([0,1]^{2}\right) \subset R^{2}$ and the set of functions $G=\{x(\cdot) \mid x: \Omega \rightarrow M\}$. One can verify that the functional

$$
A(x(\cdot))=\int_{C} a(t, x(t)) d t^{1}+a(t, x(t)) d t^{2}, x(\cdot) \in G
$$

is $(\rho, 1)$-geodesic quasiinex for $\rho \leq 0$ at the point $x^{0}(\cdot) \in G$ with respect to the application

$$
\eta(t)=\left(\eta^{1}(t), \eta^{2}(t)\right)=\left(A(x(t))-A\left(x^{0}(t)\right)\right)\left(\frac{\partial a}{\partial x^{1}}\left(t, x^{0}(t)\right), \frac{\partial a}{\partial x^{2}}\left(t, x^{0}(t)\right)\right) .
$$

Indeed, implication by Definition 4(i) naturally gives $A(x(\cdot)) \leq A\left(x^{0}(\cdot)\right) \Rightarrow$

$$
\int_{C}\left(\eta_{i} \frac{\partial X_{\alpha}}{\partial x^{i}}\left(t, x^{0}(t)\right)\right) d t^{\alpha} \leq 0, \quad i, \alpha=1,2
$$

which implies

$$
\begin{gathered}
\int_{C}\left(\eta_{1} \frac{\partial X_{1}}{\partial x^{1}}(t, \ldots)+\eta_{2} \frac{\partial X_{1}}{\partial x^{2}}(t, \ldots)\right) d t^{1}+\left(\eta_{1} \frac{\partial X_{2}}{\partial x^{1}}(t, \ldots)+\eta_{2} \frac{\partial X^{2}}{\partial x^{2}}(t, \ldots) d t^{2}\right) \leq 0 \\
\int_{C}\left(\eta_{1} \frac{\partial a}{\partial x^{1}}(t, \ldots)+\eta_{2} \frac{\partial a}{\partial x^{2}}(t, \ldots)\right) d t^{1}+\left(\eta_{1} \frac{\partial a}{\partial x^{1}}(t, \ldots)+\eta_{2} \frac{\partial a}{\partial x^{2}}(t, \ldots) d t^{2}\right) \leq 0
\end{gathered}
$$

By replacing $\eta(t)$, we obtain

$$
A\left(x(\cdot)-A\left(x^{0}(\cdot)\right) \int_{C}\left[\left(\frac{\partial a}{\partial x^{1}}\right)^{2}+\left(\frac{\partial a}{\partial x^{2}}\right)^{2}\right]\left(d t^{1}+d t^{2}\right) \leq 0,\right.
$$

or

$$
\left(A(x(\cdot))-A\left(x^{0}(\cdot)\right)\right) \int_{0}^{1}\left[\left(\frac{\partial a}{\partial x^{1}}\right)^{2}+\left(\frac{\partial a}{\partial x^{2}}\right)^{2}\right]_{\substack{t^{1}=\tau \\ t^{2}=\tau^{3}}}\left(1+3 \tau^{2}\right) d \tau \leq 0
$$


or $A(x(\cdot))-A\left(x^{0}(\cdot)\right) \leq 0$, which is true. In the particular case

$$
a(t, x(t))=x^{1}(t)+x^{2}(t)+t^{1}+t^{2}, x^{1}(t)=t^{1}+t^{2}, x^{2}(t)=t^{1}+t^{2},
$$

we ascertain that the differential

$$
d U(t)=a(t, x(t)) d t^{1}+a(t, x(t)) d t^{2}
$$

is closed $\left(\partial U / \partial t^{2}=\partial U / \partial t^{1}\right)$. Then, $C$ can be an arbitrary $C^{1}$-class curve joining the points $\left(t^{1}(0), t^{2}(0)\right)$ and $\left(t^{1}(1), t^{2}(1)\right)$ in $\Omega=[0,1]^{2}$.

Remark 1. This paper uses functionals of the form $A(x(\cdot))=\int_{C} X_{\alpha}\left(j_{t}^{1} x\right) d t^{\alpha}$, where $d U(t)=X_{\alpha}\left(j_{t}^{1} x\right) d t^{\alpha}$ is a closed differential, to establish the sufficient optimality conditions (in Sections 5 and 6).

\section{Necessary Optimality Conditions for Scalar Variational Problem}

Consider the following scalar multitime variational problem with constraints:

$$
\left\{\begin{array}{l}
\min S(x(\cdot)) \\
\text { subject to } \\
g\left(j_{t}^{1} x\right) \leq 0, h\left(j_{t}^{1} x\right)=0 \\
t \in C \subset \Omega, x(\cdot) \subset F(\Omega, M), x\left(t_{0}\right)=x_{0}, x\left(t_{1}\right)=x_{1}
\end{array}\right.
$$

The domain of this problem is the set

$$
D=\left\{x(\cdot) \subset F(\Omega, M) \mid g\left(j_{t}^{1} x\right) \leq 0, h\left(j_{t}^{1} x\right)=0, t \in C \subset \Omega, x(C) \subset M\right\}
$$

Suppose that $x^{0}(\cdot) \in D$ is an optimal solution to the variational problem (SVP) and that we choose the functions (Lagrange multipliers): $\lambda^{\beta}(t) \geq 0, t \in \Omega, \beta=1, \ldots, m$, such that $\lambda^{\beta}(t) g_{\beta}\left(g_{t}^{1} x^{0}\right)=0$ and $\mu^{\kappa}(t) \in R, \kappa=1, \ldots, q, t \in \Omega$. We also have $\mu^{\kappa}(t) h_{\kappa}\left(j_{t}^{1} x^{0}\right)=0$. By summing these equalities and integrating, we obtain

$$
\int_{C}\left(\lambda^{\beta}(t) g_{\beta}\left(j_{t}^{1} x^{0}\right)+\mu^{\theta}(t) h_{\theta}\left(j_{t}^{1} x^{0}\right)\right) d t^{\alpha}=0, \alpha=1, \ldots, p
$$

By writing relation (2) at the current point $x(t)\left(x(t) \in N\right.$ where $N$ is a neighborhood of $\left.x^{0}(t)\right)$ we obtain

$$
\int_{C}\left(\lambda^{\beta}(t) g_{\beta}\left(j_{t}^{1} x\right)+\mu^{\theta}(t) h_{\theta}\left(j_{t}^{1} x\right)\right) d t^{\alpha}=0, \alpha=1, \ldots, p .
$$

By summing relations (1) and (3) we obtain

$$
S(x)=\int_{C}\left[s_{\alpha}\left(j_{t}^{1} x\right)+\left(\lambda^{\beta}(t) g_{\beta}\left(j_{t}^{1}\right)+\mu^{\beta}(t) h_{\theta}\left(j_{t}^{1} x\right)\right)\right] d t^{\alpha},
$$

or simply

$$
S(x)=\int_{C} L_{\alpha}\left(j_{t}^{1} x\right) d t^{\alpha}
$$

where

$$
L_{\alpha}\left(j_{t}^{1} x\right)=s_{\alpha}\left(j_{t}^{1} x\right)+\left(\lambda^{\beta}(t) g_{\beta}\left(j_{t}^{1} x\right)+\mu^{\theta}(t) h_{\theta}\left(j_{t}^{1} x\right)\right), \alpha=1, \ldots, p .
$$

$L=\left(L_{\alpha}\left(j_{t}^{1} x\right)\right)$ is the Mond-Weir vector lagragian [3] associated with the functional $S(x)$. Udrişte $[16,17]$ considered the Lagrangian $\bar{L}=\left(\bar{L}_{\alpha}\left(j_{t}^{1} x\right)\right)$, where

$$
\bar{L}_{\alpha}\left(j_{t}^{1} x\right)=\tau s_{\alpha}\left(j_{t}^{1} x\right)+\left(\lambda^{\beta}(t) g_{\beta}\left(j_{t}^{1} x\right)+\mu^{\theta}(t) h_{\theta}\left(j_{t}^{1} x\right)\right), \tau \in R, \alpha=1, \ldots, p .
$$


For the functional

$$
\bar{S}(x)=\int_{C} \bar{L}_{\alpha}\left(j_{t}^{1} x\right) d t^{\alpha}
$$

it gives the following optimality result.

Theorem $1([16,22,25])$. If $x^{0}(\cdot) \in D$ minimizes the functional $\bar{S}(x)$ then $x^{0}$ is an optimal solution of the multitime system of equations

$$
\frac{\partial \bar{L}_{\alpha}}{\partial x^{i}}-D_{v} \frac{\partial \bar{L}_{\alpha}}{\partial x_{v}^{i}}=0, \alpha, v=1, \ldots, p, i=1, \ldots, n .
$$

where $\bar{L}_{\alpha}$ is completely integrable and satisfies the limit conditions on the boundary.

Remark 2. For $\tau=1$ we have $\bar{L}=L$; then Theorem 1 becomes true for $S(x)$.

Theorem 2. (Fritz-John conditions) If $x^{0}(\cdot) \in D$ is an optimal solution of the variational problem (SVP) then there exist the real scalar $\tau \in R$ and the piecewise smooth functions $\lambda=\left(\lambda^{\beta}(t)\right) \in R^{m}$ and $\mu=(\mu \theta(t)) \in R^{q}$ defined on $\Omega$, satisfying the following conditions:

$$
\left\{\begin{array}{l}
\tau \frac{\partial s_{\alpha}}{\partial x}\left(j_{t}^{1} x^{0}\right)+\lambda^{\beta}(t) \frac{\partial g_{\beta}}{\partial x}\left(j_{t}^{1} x^{0}\right)+\mu^{\kappa}(t) \frac{\partial h_{\kappa}}{\partial x}\left(j_{t}^{1} x^{0}\right)- \\
-D_{v}\left(\tau \frac{\partial s_{\alpha}}{\partial x_{v}}\left(j_{t}^{1} x^{0}\right)+\lambda^{\beta}(t) \frac{\partial g_{\beta}}{\partial x_{v}}\left(j_{t}^{1} x^{0}\right)+\mu^{\kappa}(t) \frac{\partial h_{\kappa}}{\partial x_{v}}\left(j_{t}^{1} x^{0}\right)\right)=0 \\
\lambda^{\beta}(t) g_{\beta}\left(j_{t}^{1} x^{0}\right)=0, \\
\tau \geq 0,\left(\lambda^{\beta}(t)\right) \geq 0, \beta=1, \ldots, m, \alpha=1, \ldots, p, t \in \Omega \cap C .
\end{array}\right.
$$

Theorem 2 is used for $\bar{L}$.

Definition 5. If $x^{0}(\cdot) \in D$ is an optimal solution to problem (SVP) and $\tau>0(\tau=1$ after a division by $\tau)$, then $x^{0}$ is called a normal solution of SVP.

Theorem 3. (Karush-Kuhn-Tucker conditions) If $x^{0}(\cdot) \in D$ is a normal solution to the variational problem (SVP) then there exist the piecewise smooth functions $\lambda=\left(\lambda^{\beta}(t)\right) \in R^{m}$ and $\mu(t)=\left(\mu^{\kappa}(t)\right) \in R^{q}$ defined on $\Omega$, satisfying the following conditions:

$$
\left\{\begin{array}{l}
\frac{\partial s_{\alpha}}{\partial x}\left(j_{t}^{1} x^{0}\right)+\lambda^{\beta}(t) \frac{\partial g_{\beta}}{\partial x}\left(j_{t}^{1} x^{0}\right)+\mu^{\kappa}(t) \frac{\partial h_{\kappa}}{\partial x}\left(j_{t}^{1} x^{0}\right)- \\
-D_{v}\left(\tau \frac{\partial s_{\alpha}}{\partial x_{v}}\left(j_{t}^{1} x^{0}\right)+\lambda^{\beta}(t) \frac{\partial g_{\beta}}{\partial x_{v}}\left(j_{t}^{1} x^{0}\right)+\mu^{k}(t) \frac{\partial h_{\kappa}}{\partial x_{v}}\left(j_{t}^{1} x^{0}\right)\right)=0 \\
\lambda^{\beta}(t) g_{\beta}\left(j_{t}^{1} x^{0}\right)=0, \\
(\tau=1),\left(\lambda^{\beta}(t)\right) \geq 0, \beta=1, \ldots, m, t \in \Omega \cap C .
\end{array}\right.
$$

Theorem 1 is used for $\mathrm{L}$.

\section{Necessary Efficiency Conditions for Vector Variational Curvilinear Problem}

Consider the vector curvilinear functional

$$
F(x(\cdot))=\left(\int_{C} f_{\alpha}^{1}\left(j_{t}^{1} x\right) d t^{\alpha}, \ldots, \int_{C} f_{\alpha}^{p}\left(j_{t}^{1} x\right) d t^{\alpha}\right)
$$

and the vector curvilinear integral variational problem 
$(V V C P)$

$$
\left\{\begin{array}{l}
\min F(x) \\
\text { subject to } \\
g\left(j_{t}^{1} x\right) \leq 0, h\left(j_{t}^{1} x\right)=0, t \in \Omega \cap C \\
x(\cdot) \in F(\Omega, M), x\left(t_{0}\right)=x_{0}, x\left(t_{1}\right)=x_{1} .
\end{array}\right.
$$

The domain of (VVCP) is also the set $D$.

Definition 6. A point $x^{0}(\cdot) \in D$ is an efficient solution (Pareto minimum point) of problem (VVCP) if there exist no $x(\cdot) \in D$ such that $F(x(\cdot)) \leq F\left(x^{0}(\cdot)\right)$.

Theorem 4. (Necessary efficiency conditions for $(V V C P)$ ) Let $x^{0}(\cdot) \in D$ be an efficient solution of $(V V C P)$. Then, there are a vector $\tau=\left(\tau_{r}\right) \in R^{p}$ and piecewise smooth functions $\lambda(t)=\left(\lambda^{\beta}(t)\right) \in R^{m}$ and $\mu(t)=$ $\left(\mu^{\kappa}(t)\right) \in R^{q}$ defined on $\Omega$ that satisfy the conditions:

$$
\left\{\begin{array}{l}
\tau_{r} \frac{\partial f_{\alpha}^{r}}{\partial x}+\lambda^{\beta}(t) \frac{\partial g_{\beta}}{\partial x}+\mu^{\kappa}(t) \frac{\partial h_{\kappa}}{\partial x}- \\
-D_{v}\left(\tau_{r} \frac{\partial f_{\alpha}^{r}}{\partial x_{v}}+\lambda^{\beta}(t) \frac{\partial g_{\beta}}{\partial x_{v}}+\mu^{\kappa}(t) \frac{\partial h_{\kappa}}{\partial x_{v}}\right)=0 \\
\lambda^{\beta}(t) g_{\beta}\left(j_{t}^{1} x^{0}\right)=0, \beta=1, \ldots, m \\
\left(\tau_{r}\right) \geq 0,\left(\lambda^{\beta}(t)\right) \geq 0, r=1, \ldots, p, \alpha=1, \ldots, p, \kappa=1, \ldots, q, t \in \Omega \cap C .
\end{array}\right.
$$

where $\frac{\partial f_{\alpha}^{r}}{\partial x}=\frac{\partial f_{\alpha}^{r}}{\partial x}\left(j_{t}^{1} x^{0}\right)$.

Proof of Theorem 4. If $x^{0}(\cdot) \in D$ is an efficient solution of (VVCP) the inequality $F(x(\cdot)) \leq F\left(x^{0}(\cdot)\right)$, $\forall x \in D$ is false. Then, there exist $r \in\{1, \ldots, p\}$ and a neighborhood $N$ in $D$ of $x^{0}(\cdot)$ such that $F_{r}(x(\cdot)) \geq$ $F_{r}\left(x^{0}(\cdot)\right), \forall x \in N$. Therefore, $x^{0}(\cdot)$ is an optimal solution to the following variational problem:

$(\operatorname{SVCP})_{\alpha}$

$$
\left\{\begin{array}{l}
\min F_{r}(x(\cdot))=\int_{C} f_{\alpha}^{r}\left(j_{t}^{1} x\right) d t^{\alpha} \\
\text { subject to } \\
g\left(j_{t}^{1} x\right) \leq 0, h\left(j_{t}^{1} x\right)=0, \\
x(\cdot) \in N, x\left(t_{0}\right)=x_{0}, x\left(t_{1}\right)=x_{1} .
\end{array}\right.
$$

According to Theorem 2, there are a scalar $\theta^{r} \in R$ and piecewise smooth functions $\lambda_{r}^{\beta}(t) \in R$ and $\mu_{r}^{\kappa}(t) \in R$ such that the following conditions are satisfied:

$$
\left\{\begin{array}{l}
\theta^{r} \frac{\partial f_{\alpha}^{r}}{\partial x}+\lambda_{r}^{\beta}(t) \frac{\partial g_{\beta}}{\partial x}+\mu_{r}^{\kappa}(t) \frac{\partial h_{\kappa}}{\partial x}- \\
-D_{v}\left(\theta_{r} \frac{\partial f_{\alpha}^{r}}{\partial x_{v}}+\lambda_{r}^{\beta}(t) \frac{\partial g_{\beta}}{\partial x_{v}}+\mu_{r}^{\kappa}(t) \frac{\partial h_{\kappa}}{\partial x_{v}}\right)=0 \\
\lambda^{\beta}(t) g_{\beta}\left(j_{t}^{1} x^{0}\right)=0 \\
\theta^{r} \geq 0, \lambda_{r}^{\beta}(t) \geq 0, \beta=1, \ldots, m, t \in \Omega
\end{array}\right.
$$

where $\frac{\partial f_{\alpha}^{r}}{\partial x}=\frac{\partial f_{\alpha}^{r}}{\partial x}\left(j_{t}^{1} x^{0}\right)$.

We denote $S=\sum_{r=1}^{p} \theta^{r}$ and

$$
\tau_{r}=\left\{\begin{array}{lll}
\frac{\theta^{r}}{S}, & \text { if } \quad F_{r}(x(\cdot)) \geq F_{r}\left(x^{0}(\cdot)\right), \quad r=1, \ldots, p \\
0, & \text { if } \quad F_{r}(x(\cdot))<F_{r}\left(x^{0}(\cdot)\right) .
\end{array}\right.
$$


We also define $\lambda_{r}^{\beta}(t)=0$ and $\mu_{r}^{\kappa}(t)=0$ for all $F_{r}(x(\cdot))<F_{r}\left(x^{0}(\cdot)\right)$.

Now, we sum the first relation of (4) after $r=1, \ldots, p$ and make the $\tau=\left(\tau_{1}, \ldots, \tau_{p}\right)^{\prime}$,

$$
\lambda^{\beta}(t)=\left(\sum_{r=1}^{p} \lambda_{r}^{\beta}(t)\right) / S, \mu^{\kappa}(t)=\left(\sum_{r=1}^{p} \mu_{r}^{\kappa}(t)\right) / S .
$$

Then, the relations (VFJ) are obtained.

Definition 7. The point $x^{0}(\cdot) \in D$ is called a normal efficient solution to the problem (VCP) if $\tau \neq 0$, $e^{\prime} \tau=1$, where $e=(1, \ldots, 1)^{\prime} \in R^{p}$.

\section{Necessary Efficiency Conditions for Quotient Variational Curvilinear Problem}

Consider the vector functional of ratios of integrals

$$
Q(x(\cdot))=\left(\frac{\int_{C} f_{\alpha}^{1}\left(j_{t}^{1} x\right) d t^{\alpha}}{\int_{C} \kappa_{\alpha}^{1}\left(j_{t}^{1} x\right) d t^{\alpha}}, \ldots, \frac{\int_{C} f_{\alpha}^{p}\left(j_{t}^{1} x\right) d t^{\alpha}}{\int_{C} k_{\alpha}^{p}\left(j_{t}^{1} x\right) d t^{\alpha}}\right)
$$

and the vector curvilinear integral variational problem

$(\mathrm{QVCP})$

$$
\left\{\begin{array}{l}
\min Q(x(\cdot)) \\
\text { subject to } \\
g\left(j_{t}^{1} x\right) \leq 0, h\left(j_{t}^{1} x\right)=0, t \in \Omega \cap C \\
x(\cdot) \in F(\Omega, M), x\left(t_{0}\right)=x_{0}, x\left(t_{1}\right)=x_{1} .
\end{array}\right.
$$

The domain of (QVCP) is also the set $D$.

Definition 8. A point $x^{0}(\cdot) \in D$ is said to be an efficient solution to (QVCP) if there is no $x(\cdot) \in D, x \neq x^{0}$, such that $Q(x(\cdot)) \leq Q\left(x^{0}(\cdot)\right)$.

We now give the necessary efficiency conditions for (QVCP). We define

$$
R^{r}(x)=\frac{\int_{C} f_{\alpha}^{r}\left(j_{t}^{1} x\right) d t^{\alpha}}{\int_{C} \kappa_{\alpha}^{r}\left(j_{t}^{1} x\right) d t^{\alpha}}, \quad r=1, \ldots, p
$$

and consider the problem

$(F P)_{r}$

$$
\left\{\begin{array}{l}
\min _{x} \frac{\int_{C} f_{\alpha}^{r}\left(j_{t}^{1} x\right) d t^{\alpha}}{\int_{C} k_{\alpha}^{r}\left(j_{t}^{1} x\right) d t^{\alpha}} \\
\text { subject to } \\
g\left(j_{t}^{1} x\right) \leq 0, h\left(j_{t}^{1} x\right)=0, \\
\frac{\int_{C} f_{\alpha}^{j}\left(j_{t}^{1} x\right) d t^{\alpha}}{\int_{C} \kappa_{\alpha}^{j}\left(j_{t}^{1} x\right) d t^{\alpha}} \leq R^{j}\left(x^{0}\right), j=1, \ldots, p, j \neq r . \\
x(t) \in F(\Omega, M), x\left(t_{0}\right)=x_{0}, x\left(t_{1}\right)=x_{1} .
\end{array}\right.
$$


We also consider the following two problems:

$(F R P)_{r}$

$$
\left\{\begin{array}{l}
\min _{x} \frac{\int_{C} f_{\alpha}^{r}\left(j_{t}^{1} x\right) d t^{\alpha}}{\int_{C} k_{\alpha}^{r}\left(j_{t}^{1} x\right) d t^{\alpha}}\left[=R^{r}\left(x^{0}\right)\right] \\
\text { subject to } \\
g\left(j_{t}^{1} x\right) \leq 0, h\left(j_{t}^{1} x\right)=0, \\
\int_{C}\left[f_{\alpha}^{j}\left(j_{t}^{1} x\right)-R^{j}\left(x^{0}\right) \kappa_{\alpha}^{j}\left(j_{t}^{1} x\right)\right] d t^{\alpha}, j=1, \ldots p, j \neq r, \\
x(t) \in F(\Omega, M), x\left(t_{0}\right)=x_{0}, x\left(t_{1}\right)=x_{1} .
\end{array}\right.
$$

and

$(S R P)_{r}$

$$
\left\{\begin{array}{l}
\min _{x} \int_{C}\left[f_{\alpha}^{r}\left(j_{t}^{1} x\right)-R^{r}\left(x^{0}\right) \kappa_{\alpha}^{r}\left(j_{t}^{1} x\right)\right] d t^{\alpha} \\
\text { subject to } \\
g\left(j_{t}^{1} x\right) \leq 0, h\left(j_{t}^{1} x\right)=0, \\
\int_{C}\left[f_{\alpha}^{j}\left(j_{t}^{1} x\right)-R^{j}\left(x^{0}\right) \kappa_{\alpha}^{j}\left(j_{t}^{1} x\right)\right] d t^{\alpha}, j=1, \ldots, p, j \neq r, \\
x(t) \in F(\Omega, M), x\left(t_{0}\right)=x_{0}, x\left(t_{1}\right)=x_{1} .
\end{array}\right.
$$

Lemma $1([10]) \cdot x^{0}(\cdot) \in D$ is optimal to $(F R P)$ if and only if $x^{0}(\cdot)$ is optimal to (SRP).

Definition 9. A point $x^{0}(\cdot) \in D$ is called a normal efficient solution to (QVCP) if it is an efficient solution to this problem and if it is an optimal point to at least one of the scalar problems $(S R P)_{r}, r=1 \ldots, p$.

Now, we prove the main problem of the paper.

Theorem 5. (Necessary efficiency in $(Q V C P))$ Let $x^{0}(\cdot) \in D$ be a normal efficient solution of problem (QVCP). Then there exist a vector $\tau=\left(\tau_{r}\right) \in R^{p}$ and the piecewise smooth functions $\lambda=\left(\lambda^{\beta}(t)\right) \in R^{m}$ and $\mu=\left(\mu^{\kappa}(t)\right) \in R^{q}$ defined on $\Omega$ that satisfy the conditions:

$$
\left\{\begin{array}{l}
\tau_{r}\left[\frac{\partial f_{\alpha}^{r}}{\partial x}-R^{r}\left(x^{0}\right) \frac{\partial \kappa_{\alpha}^{r}}{\partial x}\right]+\lambda^{\beta}(t) \frac{\partial g_{\beta}}{\partial x}+\mu^{\kappa}(t) \frac{\partial h_{\kappa}}{\partial x}- \\
-D_{v}\left(\tau_{r}\left[\frac{\partial f_{\alpha}^{r}}{\partial x_{v}}-R^{r}\left(x^{0}\right) \frac{\partial \kappa_{\alpha}^{r}}{\partial x_{v}}\right]+\lambda^{\beta}(t) \frac{\partial g_{\beta}}{\partial x_{v}}+\mu^{\kappa}(t) \frac{\partial h_{\kappa}}{\partial x_{v}}\right)=0 \\
\lambda^{\beta}(t) g_{\beta}\left(j_{t}^{1} x^{0}\right)=0, \beta=1, \ldots, m, \\
\tau \geq 0, \lambda(t) \geq 0, r=1, \ldots, p, \kappa=1, \ldots, q, t \in \Omega .
\end{array}\right.
$$

where $\frac{\partial f_{\alpha}^{r}}{\partial x}=\frac{\partial f_{\alpha}^{r}}{\partial x}\left(j_{t}^{1} x^{0}\right)$

Proof of Theorem 5. According to Definitions 5 and 7, there exists $r \in\{1, \ldots, p\}$ such that $x^{0}(\cdot)$ is an optimal point to the scalar problem (SRP) $r$. Then, the proof of the theorem is similar to the one of Theorem 4 , where for $r=1, \ldots, p, f_{\alpha}^{r}\left(j_{t}^{1} x\right)$ is replaced by $f_{\alpha}^{r}\left(j_{t}^{1} x\right)-R^{r}\left(x^{0}\right) k_{\alpha}^{r}\left(j_{t}^{1} x\right)$.

Theorem 6. (Necessary efficiency in $(Q V C P)$ ) Let $x^{0}(\cdot) \in D$ be a normal efficient solution to problem $(Q V C P)$. Then, there exist a vector $\tau=\left(\tau_{r}\right) \in R^{p}$ and piecewise smooth functions $\lambda=\left(\lambda^{\beta}(t)\right) \in R^{m}$ and $\mu=\left(\mu^{\kappa}(t)\right) \in R^{q}$ defined on $\Omega$ that satisfy the conditions:

$$
\left\{\begin{array}{l}
\tau_{r}\left[K^{r}\left(x^{0}\right) \frac{\partial f_{\alpha}^{r}}{\partial x}-F^{r}\left(x^{0}\right) \frac{\partial \kappa_{\alpha}^{r}}{\partial x}\right]+\lambda^{\beta}(t) \frac{\partial g_{\beta}}{\partial x}+\mu^{\kappa}(t) \frac{\partial h_{\kappa}}{\partial x}- \\
-D_{v}\left(\tau_{r}\left[K^{r}\left(x^{0}\right) \frac{\partial f_{\alpha}^{r}}{\partial x_{v}}-F^{r}\left(x^{0}\right) \frac{\partial \frac{\gamma}{\alpha}_{\alpha}^{r}}{\partial x_{v}}\right]+\lambda^{\beta}(t) \frac{\partial g_{\beta}}{\partial x_{v}}+\mu^{\kappa}(t) \frac{\partial h_{k}}{\partial x_{v}}\right)=0 \\
\lambda^{\beta}(t) g_{\beta}\left(j_{t}^{1} x^{0}\right)=0, \beta=1, \ldots, m, \\
\tau \geq 0, \lambda(t) \geq 0, r=1, \ldots, p, \kappa=1, \ldots, q, t \in \Omega .
\end{array}\right.
$$


where $\frac{\partial f_{\alpha}^{r}}{\partial x}=\frac{\partial f_{\alpha}^{r}}{\partial x}\left(t, x^{0}, x_{v}^{0}(t)\right)$

Proof of Theorem 6. In Theorem 5 we replaced $R^{r}\left(x^{0}\right)=F^{r}\left(x^{0}(\cdot)\right) / K^{r}\left(x^{0}(\cdot)\right), r=1, \ldots, p$, where $F^{r}\left(x^{0}(\cdot)\right)=\int_{C} f_{\alpha}^{r}\left(j_{t}^{1} x\right) d t^{\alpha}$ and $K^{r}\left(x^{0}(\cdot)\right)=\int_{C} k_{\alpha}^{r}\left(j_{t}^{1} x\right) d t^{\alpha}$; then, we denote the multipliers $\lambda^{\beta}(t)$ and $\mu_{\kappa}(t)$.

Remark 3. If $x^{0}(\cdot) \in D$ is a normal efficient solution to (QVCP), then in relations (MFJ) and $(M F J)_{0}$, we have $\tau \geq 0$ with $e^{\prime} \tau=1$.

\section{Sufficient Efficiency Conditions for (VVCP) and (SCP)}

In the following, we establish the sufficient efficiency conditions for variational problems with curvilinear integrals.

main condition: Suppose that the subset $G \subset F(\Omega, M)$ is an $\eta$-geodesic invex set, where the $C^{1}$ vector function $\eta(t)$ is as in Definition 3 and $G \supset D$. Furthermore, suppose that the differential

$$
d U=\frac{\partial}{\partial t^{v}}\left(\eta_{i}(t) \frac{\partial L_{\alpha}}{\partial x_{v}^{i}}\right) d t^{\alpha}
$$

is a closed Lagrange 1-form whose primitive $U(t)$ satisfies the condition $U\left(t_{1}\right) \leq U\left(t_{2}\right)$.

For a fixed $x^{0}(\cdot) \in G$, we denote $x(\cdot) \in G$ as its geodesic perturbation.

Theorem 7. (Sufficient efficiency for $(V V C P)$ ) Let $x^{0}(\cdot) \in G, \tau=\left(\tau_{r}\right), \lambda=\left(\lambda^{\alpha}\right)$ and $\mu=\left(\mu^{\kappa}\right)$ satisfy the relations (VFJ) from Theorem 4 , let $x(\cdot) \in G$ be arbitrary and the main condition be satisfied. We consider the vector function $\eta(t)=\left(\eta_{1}(t), \ldots, \eta_{n}(t)\right) \in T M$ of $C^{1}$ class (as in Definition 3 ) and suppose that the following conditions are fulfilled:

(a) For each $r=1, \ldots, p, \int_{C} f_{\alpha}^{r}\left(j_{t}^{1} x\right) d t^{\alpha}$ is $\left(\rho_{1}^{r}, b\right)$-geodesic quasiinvex at $x^{0}(\cdot)$ with respect to $\eta$ and $d$.

(b) $\int_{C} \lambda^{\beta}(t) g_{\beta}\left(j_{t}^{1} x\right) d t^{\alpha}$ is $\left(\rho_{2}, b\right)$-geodesic quasiinvex at $x^{0}(\cdot)$ with respect to $\eta$ and $d$, for $\alpha=1, \ldots, p$. (c) $\int_{C} \mu^{\kappa}(t) h_{\kappa}\left(j_{t}^{1} x\right) d t^{\alpha}$ is monotonic $\left(\rho_{3}, b\right)$-geodesic quasiinvex at $x^{0}(\cdot)$ with respect to $\eta$ and $d$, for $\alpha=$

(d) One of the integrals of $(a),(b)$ is strictly $(\rho, b)$-geodesic quasiinvex at $x^{0}(\cdot)$.

(e) $\tau_{r} \rho_{1}^{r}+\rho_{2}+\rho_{3} \geq 0\left(\rho_{1}^{r}, \rho_{2}, \rho_{3} \in \mathbf{R}\right)$.

Then $x^{0}$ is an efficiency solution to $(V V C P)$.

Proof of Theorem 7. Let us suppose a contradiction. If $x^{0}(\cdot)$ is not an efficient solution for (VVCP); then, for each $r=1 \ldots, p$, there exists $x(\cdot) \in D\left(x \neq x^{0}\right)$, a feasible solution to (VVCP), such that

$$
\int_{C} f_{\alpha}^{r}\left(j_{t}^{1} x\right) d t^{\alpha} \leq \int_{C} f_{\alpha}^{r}\left(j_{t}^{1} x^{0}\right) d t^{\alpha}
$$

According to (a) it follows that:

$$
b\left(x, x^{0}\right) \int_{C}\left[\eta_{i}(t) \frac{\partial f_{\alpha}^{r}}{\partial x^{i}}\left(j_{t}^{1} x^{0}\right)+\frac{\partial \eta_{i}}{\partial t^{\nu}} \frac{\partial f_{\alpha}^{r}}{\partial x_{v}^{i}}\left(j_{t}^{1} x^{0}\right)\right] d t^{\alpha} \leq-\rho_{1}^{r} b\left(x, x^{0}\right) d^{2}\left(x, x^{0}\right),
$$

(according to Definition 3).

By multiplying this inequality by $\tau^{r} \geq 0$ and summing over $r=1, \ldots, p$, we obtain

$$
\begin{gathered}
b\left(x, x^{0}\right) \int_{C}\left[\eta_{i}(t) \tau^{r} \frac{\partial f_{\alpha}^{r}}{\partial x^{i}}\left(j_{t}^{1} x^{0}\right)+\frac{\partial \eta_{i}}{\partial t^{\nu}} \tau_{r} \frac{\partial f_{\alpha}^{r}}{\partial x_{v}^{i}}\left(j_{t}^{1} x^{0}\right)\right] d t^{\alpha} \leq \\
-\left(\tau_{r} \rho_{1}^{r}\right) b\left(x, x^{0}\right) d^{2}\left(x, x^{0}\right) .
\end{gathered}
$$


From the continuity of the functions we choose $x \in D\left(x \neq x^{0}\right)$ such that

$$
\left.\int_{C} \lambda^{\beta}(t) g_{\beta}\left(j_{t}^{1} x\right) d t^{\alpha} \leq \int_{C} \lambda^{\beta}(t) g_{\beta}\left(j_{t}^{1} x^{0}\right) d t^{\alpha} \quad \text { (for each } \alpha=1, \ldots, p\right) .
$$

Then, taking into account condition (b) and Definition 3, this inequality implies

$$
\begin{gathered}
b\left(x, x^{0}\right) \int_{C}\left[\eta_{i}(t) \lambda^{\beta}(t) \frac{\partial g_{\beta}}{\partial x^{i}}\left(j_{t}^{1} x^{0}\right)+\frac{\partial \eta_{i}}{\partial t^{\nu}} \lambda^{\beta}(t) \frac{\partial g_{\beta}}{\partial x_{v}^{i}}\left(j_{t}^{1} x^{0}\right)\right] d t^{\alpha} \leq \\
-\rho_{2} b\left(x, x^{0}\right) d^{2}\left(x, x^{0}\right),
\end{gathered}
$$

where $\alpha=1, \ldots, p$.

Taking into account condition (c) and Definition 4 , for each $\alpha=1, \ldots, p$, from

$$
\int_{C} \mu^{\kappa}(t) h_{\kappa}\left(j_{t}^{1} x\right) d t^{\alpha}=\int_{C} \mu^{\kappa}(t)^{\prime} h_{\kappa}\left(j_{t}^{1} x^{0}\right) d t^{\alpha}
$$

we obtain

$$
\begin{gathered}
b\left(x, x^{0}\right) \int_{C}\left[\eta_{i}(t) \mu^{\kappa}(t) \frac{\partial h_{\kappa}}{\partial x^{i}}\left(j_{t}^{1} x^{0}\right)+\frac{\partial \eta_{i}}{\partial t^{\nu}} \mu^{\kappa}(t) \frac{\partial h_{\kappa}}{\partial x_{v}^{i}}\left(j_{t}^{1} x^{0}\right)\right] d t^{\alpha} \leq \\
-\rho_{3} b\left(x, x^{0}\right) d^{2}\left(x, x^{0}\right),
\end{gathered}
$$

By summing relations (5), (6) and (7) and taking into account (d), for $x \in D$, we obtain

$$
\begin{aligned}
& \int_{C} \eta_{i}(t)\left[\tau_{r} \frac{\partial f_{\alpha}^{r}}{\partial x^{i}}+\lambda^{\beta} \frac{\partial g_{\beta}}{\partial x^{i}}+\mu^{\kappa}(t) \frac{\partial h_{\kappa}}{\partial x^{i}}\right]\left(j_{t}^{1} x^{0}\right) d t^{\alpha}+ \\
& +b\left(x, x^{0}\right) \int_{C} \frac{\partial \eta_{i}}{\partial t^{v}}\left[\tau_{r} \frac{\partial f_{\alpha}^{r}}{\partial x_{v}^{i}}+\lambda^{\beta}(t) \frac{\partial g_{\beta}}{\partial x_{v}^{i}}+\mu^{\kappa}(t) \frac{\partial h_{\kappa}}{\partial x_{v}^{i}}\right]\left(j_{t}^{1} x^{0}\right) d t^{\alpha}< \\
& <-\left(\tau_{r} \rho_{1}^{r}+\rho_{2}+\rho_{3}\right) b\left(x, x^{0}\right) d^{2}\left(x, x^{0}\right) .
\end{aligned}
$$

From (8), it results that $b\left(x, x^{0}\right)$ and

$$
\begin{aligned}
& \int_{C} \eta_{i}(t)\left[\tau_{r} \frac{\partial f_{\alpha}^{r}}{\partial x^{i}}+\lambda^{\beta}(t) \frac{\partial g_{\alpha}}{\partial x^{i}}+\mu^{\beta}(t) \frac{\partial h_{\beta}}{\partial x^{i}}\right]\left(j_{t}^{1} x^{0}\right) d t^{\alpha}+ \\
& +\int_{C} \frac{\partial \eta_{i}}{\partial t^{v}}\left[\tau_{r} \frac{\partial f_{\alpha}^{r}}{\partial x_{v}^{i}}+\lambda^{\beta}(t) \frac{\partial g_{\beta}}{\partial x_{v}^{i}}+\mu^{\kappa}(t) \frac{\partial h_{\kappa}}{\partial x_{v}^{i}}\right]\left(j_{t}^{1} x^{0}\right) d t^{\alpha}< \\
& <-\left(\tau_{r} \rho_{1}^{r}+\rho_{2}+\rho_{3}\right) d^{2}\left(x, x^{0}\right) .
\end{aligned}
$$

We denote

$$
L_{\alpha}=\left[\tau_{r} f_{\alpha}^{r}+\lambda^{\beta}(t) g_{\beta}+\mu^{\kappa}(t) h_{\kappa}\right]\left(j_{t}^{1} x^{0}\right)
$$

and then relation (9) becomes

$$
\int_{C} \eta_{i}(t) \frac{\partial L_{\alpha}}{\partial x^{i}} d t^{\alpha}+\int_{\Omega} \frac{\partial \eta_{i}}{\partial t^{v}} \frac{\partial L_{\alpha}}{\partial x_{v}^{i}} d t^{\alpha}<-\left(\tau_{r} \rho_{1}^{r}+\rho_{2}+\rho_{3}\right) d^{2}\left(x, x^{0}\right) .
$$

where we denoted

$$
\frac{\partial L_{\alpha}}{\partial x}=\frac{\partial L_{\alpha}}{\partial x}\left(j_{t}^{1} x^{0}\right), \frac{\partial L_{\alpha}}{\partial x_{v}^{i}}=\frac{\partial L_{\alpha}}{\partial x_{v}^{i}}\left(j_{t}^{1} x^{0}\right) .
$$

Integration by parts in the second integral of (10) gives

$$
\frac{\partial}{\partial t^{v}}\left(\eta_{i}(t)^{\prime} \frac{\partial L_{\alpha}}{\partial x_{v}^{i}}\right)=\frac{\partial \eta_{i}}{\partial t^{v}} \frac{\partial L_{\alpha}}{\partial x_{v}^{i}}+\eta_{i}(t) \frac{\partial}{\partial t^{v}}\left(\frac{\partial L_{\alpha}}{\partial x_{v}^{i}}\right), v=1, \ldots, m
$$

and

$$
\int_{C} \frac{\partial \eta_{i}}{\partial t^{\nu}} \frac{\partial L_{\alpha}}{\partial x_{v}^{i}} d t^{\alpha}=\int_{C} \frac{\partial}{\partial t^{\nu}}\left(\eta_{i}(t) \frac{\partial L_{\alpha}}{\partial x_{v}^{i}}\right) d t^{\alpha}-\int_{C} \eta_{i}(t) \frac{\partial}{\partial t^{\nu}}\left(\frac{\partial L_{\alpha}}{\partial x_{v}^{i}}\right) d t^{\alpha}
$$


Using relation (11), relation (10) becomes

$$
\begin{gathered}
\int_{C} \eta_{i}(t)\left[\frac{\partial L_{\alpha}}{\partial x^{i}}-\frac{\partial}{\partial t^{v}}\left(\frac{\partial L_{\alpha}}{\partial x_{v}^{i}}\right)\right] d t^{\alpha}+\int_{C} \frac{\partial}{\partial t^{v}}\left(\eta_{i}(t) \frac{\partial L_{\alpha}}{\partial x_{v}^{i}}\right) d t^{\alpha}< \\
<-\left(\tau_{r} \rho_{1}^{r}+\rho_{2}+\rho_{3}\right) d^{2}\left(x, x^{0}\right) .
\end{gathered}
$$

Taking into account the first relation of (VFJ), (12) becomes

$$
\int_{C} \frac{\partial}{\partial t^{v}}\left(\eta_{i}(t) \frac{\partial L_{\alpha}}{\partial x_{v}^{i}}\right) d t^{\alpha}<-\left(\tau_{r} \rho_{1}^{r}+\rho_{2}+\rho_{3}\right) d^{2}\left(x, x^{0}\right) .
$$

According to [18], the total divergence is equal to the total derivative $d U$. Moreover, according to the main condition, $d U$ is a closed 1-form. Then, there exists the primitive $U(T)$, with $U\left(t_{1}\right) \leq U\left(t_{2}\right)$, and the integral of (13) becomes

$$
\int_{C} d U=\left.U(t)\right|_{t_{1}} ^{t_{2}}=U\left(t_{2}\right)-U\left(t_{1}\right) \geq 0
$$

Consequently, relation (13) becomes

$$
0<-\left(\tau_{r} \rho_{1}^{r}+\rho_{2}+\rho_{3}\right) d^{2}\left(x, x^{0}\right)
$$

With $d\left(x, x^{0}\right) \geq 0$ and hypothesis (e), we obtain the inequality $0<0$, which is a false. Therefore, $x^{0}(\cdot)$ is an efficient solution to (VVP).

If in Theorems 7 the integrals from hypotheses (b) and (c) are replaced by the integral $\int_{C}\left[\lambda^{\beta}(t) g_{\beta}\left(j_{t}^{1} x\right)+\mu^{\kappa}(t) h_{\kappa}\left(j_{t}^{1} x\right)\right] d t^{\alpha}$, then the following results are obtained:

Corollary 1. (Sufficient efficiency conditions for (VVCP)) Let $x^{0}(\cdot) \in G, \tau, \lambda$ and $\mu$ satisfy the relations (VFJ) from Theorem 4, let arbitrary $x(\cdot) \in D$ and let the main condition be satisfied. We consider a vector function $\eta$ as in Definition 3 and assume that the following conditions are satisfied:

$\left(a^{\prime}\right)$ For each $r=1, \ldots, p, \int_{C} f_{\alpha}^{r}\left(j_{t}^{1} x\right) d t^{\alpha}$ is $\left(\rho_{1}^{r}, b\right)$-geodesic quasiinvex at $x^{0}(\cdot)$ with respect to $\eta$ and $d$.

$\left(b^{\prime}\right) \quad \int_{C}\left[\lambda^{\beta}(t) g_{\beta}\left(j_{t}^{1} x\right)+\mu^{\kappa}(t) h_{\kappa}\left(j_{t}^{1} x\right)\right] d t^{\alpha}$ is $\left(\rho_{2}, b\right)$-geodesic quasiinvex at $x^{0}(\cdot)$ with respect to $\eta$ and $d$, for $\alpha=1, \ldots, p$.

$\left(c^{\prime}\right)$ One of the integrals of $\left(a^{\prime}\right)$ and $\left(b^{\prime}\right)$ is strictly $(\rho, b)$-geodesic quasiinvex at $x^{0}(\cdot)$ with respect to $\eta$ and $d$ $\left(\rho=\rho_{r}^{1}\right.$ or $\rho_{2}$, respectively).

(d') $\tau^{r} \rho_{r}^{1}+\rho_{2} \geq 0$.

Then $x^{0}(\cdot)$ is an efficient solution to $(V V C P)$.

Corollary 2. (Sufficient conditions for $(S C P)$ ) Let $x^{0}(\cdot) \in G, \tau, \lambda$ and $\mu$ satisfy the relations (FJ) from Theorem 1, let arbitrary $x^{0}(\cdot) \in D$, and let the main condition be satisfied. We consider a vector function $\eta$ as in Definition 3 and suppose that the following conditions are satisfied:

(a) $\quad \int_{C} s_{\alpha}\left(j_{t}^{1} x\right) d t^{\alpha}$ is $(\rho, b)$-geodesic quasiinvex at $x^{0}(\cdot)$ with respect to $\eta$ and $d$.

$(\bar{b})$ Condition $\left(b^{\prime}\right)$ from Corollary 1 is true.

$(\bar{c})$ One of the integrals of $(\bar{a})$ and $\left(b^{\prime}\right)$ from Corollary 1 is strictly $(\rho, b)$-geodesic quasiinvex at $x^{0}(\cdot)$ with respect to $\eta$ and $d$.

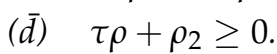

Then $x^{0}(\cdot)$ is an efficient solution to $(S C P)$. 


\section{Sufficient Efficiency Conditions for (QVCP)}

In the following, we establish the efficiency sufficient conditions for the problem (QVCP).

Theorem 8. (Sufficient efficiency for $(Q V C P))$ Let $x^{0}(\cdot) \in G, \tau=\left(\tau^{r}\right), \lambda=\left(\lambda^{\beta}(t)\right)$ and $\mu=\left(\mu^{k}(t)\right)$ satisfy the relations (MFJ) from Theorem 5 , let arbitrary $x^{0}(\cdot) \in D$ and let the main condition be satisfied. Furthermore, we consider a vector function $\eta$ as in Definition 3 and suppose that the following conditions are satisfied:

$\left(a^{\prime}\right)$ For each $r=1, \ldots, p, \int_{C}\left[f_{\alpha}^{r}\left(j_{t}^{1} x\right)-R^{r}\left(x^{0}\right) \kappa_{\alpha}^{r}\left(j_{t}^{1} x\right)\right] d t^{\alpha}$ is $\left(\rho_{i}^{1}, b\right)$-geodesic quasiinvex at $x^{0}(\cdot)$ with respect to $\eta$ and $d$.

(b') $\int_{C} \lambda^{\beta}(t)^{\prime} g_{\beta}\left(j_{t}^{1} x\right) d t^{\alpha}$ is $\left(\rho_{2}, b\right)$-geodesic quasiinvex at $x^{0}(\cdot)$ with respect to $\eta$ and $d$, for $\alpha=1, \ldots, p$.

(c') $\int_{C} \mu^{k}(t)^{\prime}\left(j_{t}^{1} x\right) d t^{\alpha}$ is $\left(\rho_{3}, b\right)$-geodesic quasiinvex at $x^{0}(\cdot)$ with respect to $\eta$ and $d$, for $\alpha=1, \ldots, p$.

$\left(d^{\prime}\right)$ One of the integrals of $\left(a^{\prime}\right),\left(b^{\prime}\right)$ and $\left(c^{\prime}\right)$ is strictly $(\rho, b)$-geodesic quasiinvex at $x^{0}(\cdot)$ with respect to $\eta$ and $d\left(\rho=\rho_{r}^{1}, \rho_{2}\right.$ or $\rho_{3}$, respectively).

(é) $\tau^{r} \rho_{r}^{1}+\rho_{2}+\rho_{3} \geq 0$.

Then $x^{0}(\cdot)$ is an efficient solution to $(Q V C P)$.

Proof of Theorem 8. The proof is similar to the one of Theorem 7 where, for each $r=1, \ldots, p, f^{r}\left(j_{t}^{1} x\right)$ is replaced by $f^{r}\left(j_{t}^{1} x\right)-R^{r}\left(x_{0}\right) k^{r}\left(j_{t}^{1} x\right)$.

Theorem 9. (Sufficient efficiency for $(Q V C P)$ ) Let $x^{0}(\cdot) \in G, \tau=\left(\tau^{r}\right), \lambda=\left(\lambda^{\beta}(t)\right)$ and $\mu=\left(\mu^{\kappa}(t)\right.$ ) satisfy the relations (MFV) from Theorem 6, let arbitrary $x(\cdot) \in D$ and assume the main condition is satisfied. Furthermore, consider a vector function $\eta$ as in Definition 3 and suppose that the following conditions are satisfied:

$\left(a^{\prime \prime}\right)$ For each $r=1, \ldots, p, \int_{C}\left[K^{r}\left(x^{0}\right) f_{\alpha}^{r}\left(j_{t}^{1} x\right)-F^{r}\left(x^{0}\right) \kappa_{\alpha}^{r}\left(j_{t}^{1} x\right)\right] d t^{\alpha}$ is $\left(\rho_{r}^{1}, b\right)$-geodesic quasiinvex at $x^{0}(\cdot)$ with respect to $\eta$ and $d$.

$\left(b^{\prime \prime}\right)\left(b^{\prime}\right),\left(c^{\prime}\right)$ and $\left(e^{\prime}\right)$ of Theorem 8.

$\left(c^{\prime \prime}\right)$ One of the integrals of $\left(a^{\prime \prime}\right),\left(b^{\prime}\right)$ and $\left(c^{\prime}\right)$ is strictly $(\rho, b)$-geodesic quasiinvex at $x^{0}(\cdot)$ with respect to $\eta$ and $d\left(\rho=\rho_{r}^{1}, \rho_{2}\right.$ or $\rho_{3}$, respectively).

Then $x^{0}(\cdot)$ is an efficient solution to $(Q V C P)$.

Proof of Theorem 9. The proof is similar to one of Theorem 8 , where hypothesis $\left(a^{\prime}\right)$ is replaced by hypothesis $\left(\mathrm{a}^{\prime \prime}\right)$ of this theorem.

Corollary 3. (Sufficient efficiency conditions for (QVCP). Let $x^{0}(\cdot) \in G, \tau, \lambda=\left(\lambda^{\beta}(t)\right)$ and $\mu=\left(\mu^{\kappa}(t)\right.$ ) satisfy the relations (MFJ) from Theorem 5, let arbitrary $x(\cdot) \in D$ and assume that the main condition is satisfied. Furthermore, consider the vector functions $\eta$ as in Definition 3 and suppose that the following conditions are satisfied:

$\left(a^{\prime}\right) \quad$ For each $r=1, \ldots, p, \int_{C}\left[f_{\alpha}^{r}\left(j_{t}^{1} x\right)-R\left(x^{0}\right) \kappa_{\alpha}^{r}\left(j_{t}^{1} x\right)\right] d t^{\alpha}$ is $\left(\rho_{r}^{1}, b\right)$-geodesic quasiinvex at $x^{0}(\cdot)$ with respect to $\eta$ and $d$.

$\left(b^{\prime}\right)$ Conditions $\left(b^{\prime}\right)$ and $\left(d^{\prime}\right)$ from Corollary 1.

$\left(c^{\prime}\right)$ One of the integrals of $\left(a^{\prime}\right)$ and $\left(b^{\prime}\right)$ is strictly $(\rho, b)$-geodesic quasiinvex at $x^{0}(\cdot)$ with respect to $\eta$ and $d$.

Then $x^{0}(\cdot)$ is an efficient solution to $(Q V C P)$.

Corollary 4. (Sufficient efficiency conditions for $(Q V C P)$ ) Let $x^{0}(\cdot) \in G, \tau, \lambda=\left(\lambda^{\beta}(t)\right)$ and $\mu=\left(\mu^{\kappa}(t)\right.$ ) satisfy the relations (MFV) from Theorem 6. Furthermore, consider a vector function $\eta$ as in Definitions 1 and 2, and assume that the following conditions are satisfied:

$\left(a^{\prime \prime}\right)$ For each $r=1 \ldots, p, \int_{C}\left[K^{r}\left(x^{0}\right) f_{\alpha}^{r}\left(j_{t}^{1} x\right)-F^{r}\left(x^{0}\right) k_{\alpha}^{r}\left(j_{t}^{1} x\right)\right] d t^{\alpha}$ is $\left(\rho_{1}^{r}, b\right)$-geodesic quasiinvex at $x(\cdot)$ with respect to $\eta$ and $d$. 
$\left(b^{\prime \prime}\right)$ Conditions $\left(b^{\prime}\right)$ and $\left(d^{\prime}\right)$ from Corollary 1.

$\left(c^{\prime \prime}\right)$ One of the integrals of $\left(a^{\prime \prime}\right)$ and $\left(b^{\prime}\right)$ is strictly $(\rho, b)$-geodesic quasiinvex at $x^{0}(\cdot)$ with respect to $\eta$ and $d$.

Then $x^{0}(\cdot)$ is an efficient solution to $(Q V C P)$.

\section{Conclusions and Further Developments}

In this paper, new classes of variational control problems of minimizing a vector of path-independent curvilinear integral (mechanical or cost) functionals ratios, were considered. Starting from scalar variational problems (SVP) elaborated by Udriste et al. $[16,17,20,22,23]$ by which optimality conditions of variational problems in the multitime approach (so called multitime variational problems) with constraints were introduced in literature, in this paper by using curvilinear integrals and generalized invex functionals, new necessary and sufficient conditions of efficiency were obtained. In particular, we have formulated and proved necessary geodesic efficiency conditions in the considered scalar, vector and vector quotient variational control problems, by using the notation of normal geodesic efficient solution and new notions of geodesic efficient solution. As well, by using the original concept of $(\rho, b)$-geodesic quasiinvexity associated with path-independent curvilinear integral functionals, sufficient conditions of geodesic efficiency for a feasible solution in the considered vector and vector quotient variational control problems have been derived. The proposed framework could be depth considering the "theory of functionals," taking into account the variational methodology so useful for the study of regularity properties of integral functionals. In this direction of ongoing research, see $[26,27]$ for more.

Author Contributions: Conceptualization, T.C. and Ş.M.; Methodology, S..M. and B.A.P.; Conceptualization, Methodology and Supervision M.F.; Writing—original draft, T.C. and B.A.P. All authors have read and agreed to the published version of the manuscript.

Funding: This research was funded by Department of Law, Economics and Human Sciences-Decisions_LAB grant number 2/2020 and the APC was funded by Piano Dipartimento di Eccellenza L.232/2016.

Acknowledgments: The authors would like to thank the anonymous referees for careful reading of the original manuscript and for several suggestions for its improvement.

Conflicts of Interest: The authors declare no conflict of interest.

\section{References}

1. Valentine, F.A. The Problem of Lagrange with Differentiable Inequality as Added Side Conditions; Contributions to the Calculus of Variations 1933-37; University of Chicago Press: Chicago, IL, USA, 1937; pp. 407-448.

2. Mond, B.; Hanson, M.A. Duality for variational problems. J. Math. Anal. Appl. 1967, 18, 355-364. [CrossRef]

3. Mond, B.; Chandra, S.; Husain, I. Duality in variational problems with invexity. J. Math. Anal. Appl. 1988, 134, 322-328. [CrossRef]

4. Mond, B.; Husain, I. Sufficient optimality criteria and duality for variational problems with generalized invexity. J. Aust. Math. Soc. Ser. B 1989, 31, 108-121. [CrossRef]

5. Preda, V. On Mond-Weir duality for variational problems. Rev. Roum. Math. Pures Appl. 1993, 38, 155-164.

6. Chankong, V.; Haimes, Y.Y. Multiobjective Decision Making: Theory and Methodology; Elsevier: New York, NY, USA, 1983.

7. Kanniappan, P. Necessary conditions for optimality of nondifferentiable convex multiobjective programming. J. Optim. Theory Appl. 1983, 40, 167-174. [CrossRef]

8. Mukherjee, R.N.; Rao, C.P. Mixed type duality for multiobjactive variational problems. J. Math. Anal. Appl. 2000, 252, 571-586. [CrossRef]

9. Preda, V.; Gramatovici, S. Some optimality conditions for class of multiobjective variational problems. Anal. Univ. Bucur. Mat. Inform. 2002, 51, 33-43.

10. Jagannathan, R. Duality for nonlinear fractional programming. Z. Oper. Res. 1973, 17, 1-3.

11. Şt. Mititelu, S. Efficiency and duality for multiobjective fractional problems in optimal control with $\rho$-quasiinvexity. In Proceedings of the ICTCAM 2007-International Conference on Trends and Challenges in Applied Mathematics, Bucharest, Romania, 20-23 June 2007. 
12. Şt. Mititelu, S. Effciency conditions for multiobjective fractional variational problems. Appl. Sci. 2008, 10, 162-175.

13. Şt. Mititelu, S.; Postolache, M. Mond-Weir dualities with Lagrangians for multiobjective fractional and non-fractional variational problems. J. Adv. Math. Stud. 2010, 3, 41-58.

14. Şt. Mititelu, S.; Stancu-Minasian, I. Efficiency and duality for multiobjective fractional and nonfractional problem with $(\rho, b)$-quasi-invexity. Yugoslav J. Oper. Res. 2009, 19, 85-99. [CrossRef]

15. Şt. Mititelu, S.; Treanţă, S. Efficiency conditions in vector control problems governed by multiple integrals. J. Appl. Math. Comput. 2018, 57, 647-665. [CrossRef]

16. Pitea, A.; Udrişte, C.; Şt. Mititelu, S. PDE-constrained optimization problems with curviliniar functional quotiens as objective vectors. Balkan J. Geom. Appl. 2009, 134, 75-88.

17. Pitea, A.; Udrişte, C.; Şt. Mititelu, S. New types dualities in PDI and PDE constrained optimization problems. J. Adv. Math. Stud. 2009, 2, 81-90.

18. Stancu-Minasian, I.M.; Şt. Mititelu, S. Multiobjective fractional variational problems with $(\rho, b)$-quasiinvexity. Proc. Rom. Acad. Ser. A 2008, 9, 5-11.

19. Kim, D.S.; Kim, A.I. Optimality and duality for nondifferentiable variational problems. J. Math. Anal. Appl. 2002, 274, 255-278. [CrossRef]

20. Udrişte, C.; Bejenaru, A. Multitime optimal control with area integral costs on boundary. Balkan J. Geom. Appl. 2011, 16, 138-154.

21. Pitea, A.; Postolache, M. Duality theorems for a new class of multitime multiobjective variational problems. J. Glob. Optim. 2012, 54, 47-58. [CrossRef]

22. Udrişte, C.; Bejenaru, A. Riemannian convexity of functionals. J. Glob. Optim. 2011, 51, 361-376. [CrossRef]

23. Udrişte, C.; Matei, L. Teorii Lagrange-Hamilton; Geometry Balkan Press: Bucuresti, Romania, 2008.

24. Barani, A.; Pouryayevali, M.R. Invex sets and preinvex functions on Riemannian manifolds. J. Math. Anal. Appl. 2007, 328, 767-779. [CrossRef]

25. Udrişte, C.; Dogaru, O.; Tevy, I. Null Lagrangian form and Euler-Lagrange PDEs. J. Adv. Math. Stud. 2008, 1, 143-156.

26. Usuba, K. Partial regularity of minimizers of $\mathrm{p}(\mathrm{x})$-growth functionals with $1<p(x)<2$. Bull. Lond. Math. Soc. 2015, 47, 455-472.

27. Goodrich, C.S.; Ragusa, M.A.; Scapellato, A. Partial regularity of solutions to p(x)-laplacian PDEs with discontinuous coefficients. J. Differ. Equ. 2020, 268, 5440-5468. [CrossRef] 